\title{
Observation of Polarization Vortices in Momentum Space
}

\author{
Yiwen Zhang, ${ }^{1,4}$ Ang Chen, ${ }^{1,4}$ Wenzhe Liu, ${ }^{1,4}$ Chia Wei Hsu, ${ }^{3}$ Bo Wang, ${ }^{1,4}$ Fang Guan, ${ }^{1,4}$ \\ Xiaohan Liu, ${ }^{1,4}$ Lei Shi, ${ }^{1,4, *}$ Ling $\mathrm{Lu},{ }^{2, \dagger}$ and Jian $\mathrm{Zi}^{1,4, \ddagger}$ \\ ${ }^{1}$ Department of Physics, Key Laboratory of Micro- and Nano-Photonic Structures (Ministry of Education), \\ and State Key Laboratory of Surface Physics, Fudan University, Shanghai 200433, China \\ ${ }^{2}$ Institute of Physics, Chinese Academy of Sciences/Beijing National Laboratory for Condensed Matter Physics, Beijing 100190, China \\ ${ }^{3}$ Department of Applied Physics, Yale University, New Haven, Connecticut 06520, USA \\ ${ }^{4}$ Collaborative Innovation Center of Advanced Microstructures, Fudan University, Shanghai 200433, China
}

(Received 2 November 2017; published 4 May 2018)

\begin{abstract}
The vortex, a fundamental topological excitation featuring the in-plane winding of a vector field, is important in various areas such as fluid dynamics, liquid crystals, and superconductors. Although commonly existing in nature, vortices were observed exclusively in real space. Here, we experimentally observed momentum-space vortices as the winding of far-field polarization vectors in the first Brillouin zone of periodic plasmonic structures. Using homemade polarization-resolved momentum-space imaging spectroscopy, we mapped out the dispersion, lifetime, and polarization of all radiative states at the visible wavelengths. The momentum-space vortices were experimentally identified by their winding patterns in the polarization-resolved isofrequency contours and their diverging radiative quality factors. Such polarization vortices can exist robustly on any periodic systems of vectorial fields, while they are not captured by the existing topological band theory developed for scalar fields. Our work provides a new way for designing high- $Q$ plasmonic resonances, generating vector beams, and studying topological photonics in the momentum space.
\end{abstract}

DOI: $10.1103 /$ PhysRevLett.120.186103

The winding of a vector field in two dimensions forms a vortex whose core is located at the field singularity, and its topological charge is defined by the quantized winding angle of the vector field [1]. Vortices are widely known in hair whorls as the winding of hair strings, in fluid dynamics as the winding of velocities, in vector beams as the winding of states of polarization [2-4], and in superconductors and superfluids as the winding of order parameters [5]. Vortices are one of the most common topological excitations in nature, but they have hardly been observed other than those in the real space. Although band degeneracies, such as Dirac cones, can be viewed as momentum-space vortices in their mathematical structures, there lacks a well-defined physical observable whose winding number is an arbitrary signed integer [6].

It was recently theoretically proposed that photons in photonic crystal slabs can support vortices with a winding far-field polarization vector in momentum space [7]; multiple vortices can exist at momentum points across the first Brillouin zone (FBZ), not necessarily at the band

Published by the American Physical Society under the terms of the Creative Commons Attribution 4.0 International license. Further distribution of this work must maintain attribution to the author(s) and the published article's title, journal citation, and DOI. edge $[8,9]$. The Bloch state at the vortex core may cease to radiate and be called a bound state in the radiation continuum [10-25]. Moreover, these momentum-space vortices could be a general phenomenon in vector fields, radiative or not. They point to a promising avenue for exploring topological properties in optics [26-31] as well as for rendering vector beams [32], but such polarization vortices have not been experimentally observed so far.

In this Letter, we experimentally demonstrate momentum-space polarization vortices in two-dimensional (2D) plasmonic crystals at visible wavelengths. The plasmonic crystals studied are flat metallic substrates coated with periodically corrugated thin dielectric layers fabricated using electron-beam lithography. The metallic substrate is a 200-nm-thick silver film evaporated on a glass substrate, and the periodic dielectric layer is a 70-nm-thick polymethyl methacrylate (PMMA) thin film (refractive index of 1.5) with a square array of cylindrical air holes (periodicity, $400 \mathrm{~nm}$; hole diameter, $290 \mathrm{~nm}$ ). Atomic force microscope images are shown in Supplemental Material [33]. The metallic film is thick enough to avoid transmission. Because of the periodicity of the PMMA array, the surface plasmon polaritons (SPPs) show well-defined band structures, and modes above the air light cone can couple into the free space and radiate [34]. Note that there is no guided mode in the wavelength region of interest. If the PMMA film is thick enough, guided modes will show a 
redshift in frequency and intersect with SPP modes, forming complex band structures of hybrid SPPs.

In Fig. 1(a), we plotted the band structure of the sample, calculated using the finite-difference-time-domain method. Vortices of the far-field polarization vectors robustly exist on each dispersion band, of which the topological charges are defined by the winding angle of the vectors divided by $2 \pi$. Here, the polarization vector is the major radiation electric-field component projected onto the lattice plane. For those vortices at all high-symmetry points in the FBZ, we provide a general way to determine the possible charges, regardless of the core degeneracy level, using mirror eigenvalues on the mirror-invariant momentum lines. The $C_{4 v}$ lattice has three mirror-invariant lines of $\Gamma-M-X-\Gamma$ in the FBZ, on which the mirror eigenvalues are \pm 1 . This means the corresponding far-field polarization vector is either parallel or perpendicular to those momentum lines. We denote these two cases as even $(+)$ and odd $(-)$ representations. By exhausting all possible combinations of representations on the high-symmetry lines surrounding each high-symmetry point, it is straightforward to see that all possible topological charges at $\Gamma$ and $M$ points are $4 n \pm 1$. Here $n$ is a signed integer. Similarly, the $X$ point has $C_{2 v}$ symmetry, and its possible charges are $2 n \pm 1$ or $2 n$, detailed in Supplemental Material [33]. These are

(a)

(b)

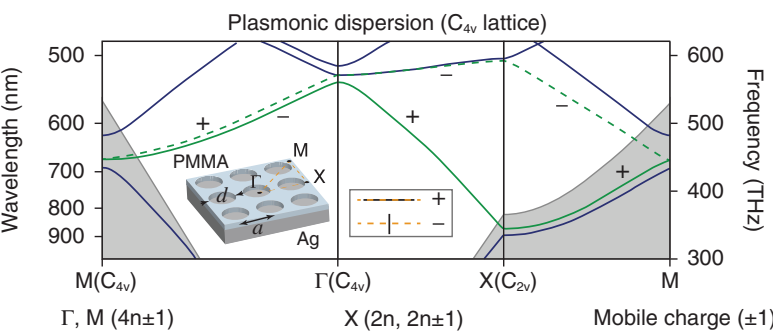

(c)
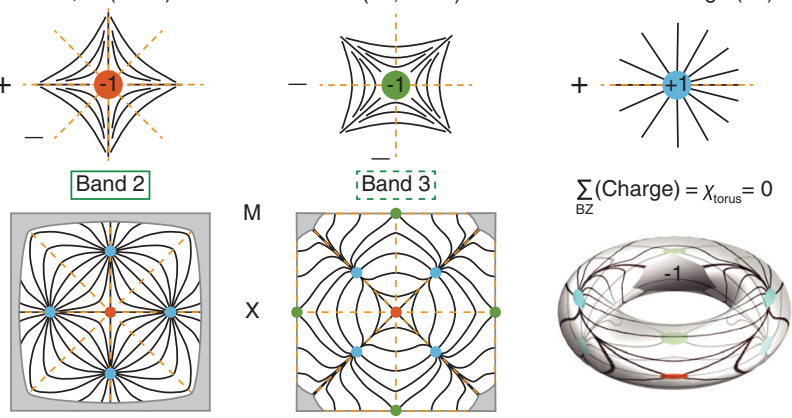

FIG. 1. (a) Simulated band structure of a plasmonic crystal with square lattice. Inset: Schematic view of the structure. The mirror eigenvalues $( \pm 1)$ of modes are labeled on the high-symmetry lines. (b) Illustration of lowest-order polarization vortices at highsymmetry momentum points. The \pm signs, the mirror eigenvalues along the surrounding high-symmetry lines; colored circles, the vortex cores and their charges. (c) Polarization vectors (represented by streamlines) plotted on the whole FBZ. The right panel shows the polarization streamlines on the 2D FBZ torus of band 3 . consistent with the previous conclusion [7]. Since one cannot distinguish the electric-field direction with its opposite due to the time-harmonic oscillations, we represent the polarization vector fields using line segments without arrows, which still allows us to precisely exhibit the vortices. We emphasize that our discussion works for both nondegenerate and degenerate states, since the representation at the vortex core is not needed for determining its charge. When the vortex core is a nondegenerate state, its polarization singularity corresponds to a nonradiative bound state in the radiation continuum (dark state). When the vortex core is a degenerate state, it can be either radiative (bright state) or nonradiative. In the degenerate and radiative case, the polarization is also ill defined at the singularity. It consists of a linear combination of all polarizations and can take any polarization in the far field.

Take bands 2 and 3, for example, whose dispersion curves are plotted in Fig. 1(a) as solid and dashed green lines, respectively. Their mirror eigenvalues [see Fig. 1(a)] on $\Gamma-M-X-\Gamma$ can be easily obtained by computing the nearfield Bloch wave functions at three points-one point on each of the three mirror-invariant momentum lines (see Supplemental Material [33]). For these two bands, the possible charges at $\Gamma$ and $M$ points are $4 n-1$, and those at the $X$ point are $2 n \pm 1$. Note that bands 2 and 3 are nondegenerate on the three momentum segments excluding the end points $(\Gamma, M$, and $X)$, so their mirror eigenvalues are constants on each momentum segment.

The sum of all vortex charges on a $2 \mathrm{D}$ dispersion band is zero, equaling to the Euler characteristic $(\chi)$ of the 2D FBZ torus. If the charge sum at high-symmetry points $\Gamma, M$, and $X$ is nonzero, there must be mobile vortices inside the FBZ to neutralize the total charge. When the system parameter is continuously tuned, the mobile vortices could potentially combine at the high-symmetry points and change their $n$ values. We computed and plotted the polarization vector fields in the whole FBZ for both bands 2 and 3 in Fig. 1(c) to illustrate our arguments. The shaded gray area indicates nonradiative states. The charges within the gray area on band 2 can be inferred to be -3 in total, and the charge at the $M$ point on band 3 can be inferred to be -1 . For the following experiments, we focus on the lowest radiative dispersion-band 2.

To experimentally characterize the band dispersions and the vortices on them, homemade polarization-resolved momentum-space imaging spectroscopy based on Fourier analysis [35] was used, as illustrated in Fig. 2(a). The working principle is the fact that the information carried by the back focal plane of an objective lens corresponds to the momentum-space (Fourier-space) information of the radiation field from the sample. There are two modes of operation. In the first mode, by positioning an imaging spectrometer, whose entrance is conjugate to the back focal plane, we map out the momentum space one line at a time within the whole visible spectrum; rotating the sample in 
plane relative to the entrance slit of the imaging spectrometer yields the entire FBZ. In the second mode, we select a wavelength with a bandpass filter (10 $\mathrm{nm}$ bandwidth) and image the isofrequency contour in the entire FBZ onto a 2D charged-coupled-device (CCD) camera in one shot; a series of filters yield a full set of isofrequency contours. More importantly, using a polarizer, we can determine the farfield states of polarization for every momentum state. Therefore, we were able to obtain the dispersion, lifetime, and polarization of nearly all the radiative states in the whole FBZ experimentally (details in Supplemental Material [33]).

Figure 2(d) shows a 3D plot of the measured band structure of the square-lattice plasmonic crystal [image in Fig. 2(b)]. The plotted data are the extinction ratio (one minus reflectivity) as a function of the wavelength and wave vector, under unpolarized illumination and averaged over two orthogonal polarizer directions at the output. The band structure is defined by the peaks in the extinction spectra, resulting from the excitations of plasmon modes. Extinction here is directly related to radiation properties of those modes. The measured band structure agrees well with the calculation in Fig. 1(a). The key feature in Fig. 2(d) is that the extinction peaks disappear at certain momentum points (marked by the blue arrows). Since dispersion bands are physically continuous, the disappearance indicates that those states cannot be excited and are decoupled from the free space with a diverging radiative quality $(Q)$ factor. Similar states were recently observed in dielectric photonic crystal slabs [15,21]. We measured the $Q$ factors of nearly all the Bloch states above the light cone by fitting the experimental extinction spectra with the temporal coupled mode theory (see Supplemental Material [33]). Figure 2(e) shows the distribution of radiative $Q$ factors on band 2 [blue dashed line in Fig. 2(d)], where a total of five vortices can be seen from the diverging $Q$. One vortex is fixed at $\Gamma$, and the other four are along the $\Gamma-X$ lines.

The extinction spectra are polarization dependent, having the relation $\mathrm{E}_{\mathrm{xt}}^{(\hat{\alpha})} \sim|\hat{t} \cdot \vec{d}|^{2}$, where $\hat{t}$ is the effective polarizer transmission axis projected onto the $\hat{s}-\hat{p}$ plane and the polarization state of the resonant radiation is defined by $\vec{d}=d_{s} \hat{s}+d_{p} \hat{p}$. (See the derivation in Supplemental Material [36].) Thus, by changing the polarizer angle $\alpha$ and measuring polarization-dependent extinction spectra, we could determine the polarization states of SPP modes. We measured extinction spectra at three polarizer angles. As shown in Fig. 2(c), the polarizer angles were $0^{\circ},-45^{\circ}$ and $90^{\circ}$ relative to the direction of the entrance slit of the spectrometer. The details of the methods are shown in Supplemental Material [36]. In general, polarization states of radiation from SPP modes are elliptical. However, due to the $180^{\circ}$ rotational symmetry $C_{2}$ of the structure, these polarization states are close to linear polarization [37]. In Fig. 2(f), we show the polarization ellipses of the far-field radiation. The green ellipses (a) Experimental setup
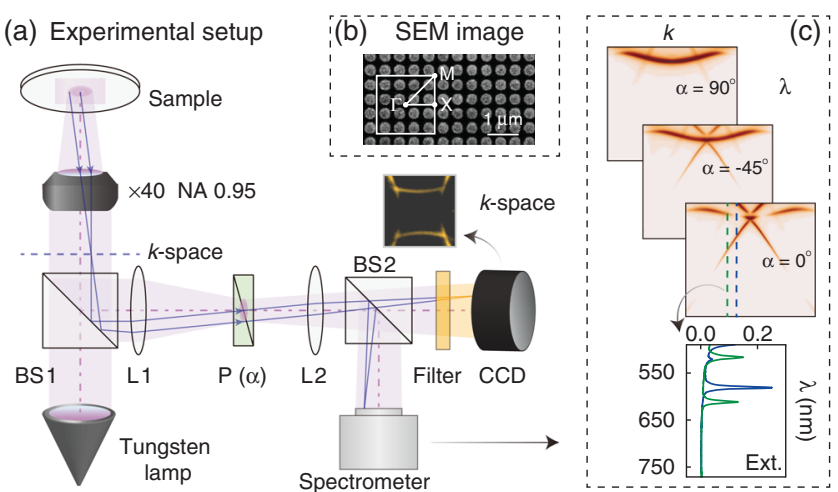

(d)
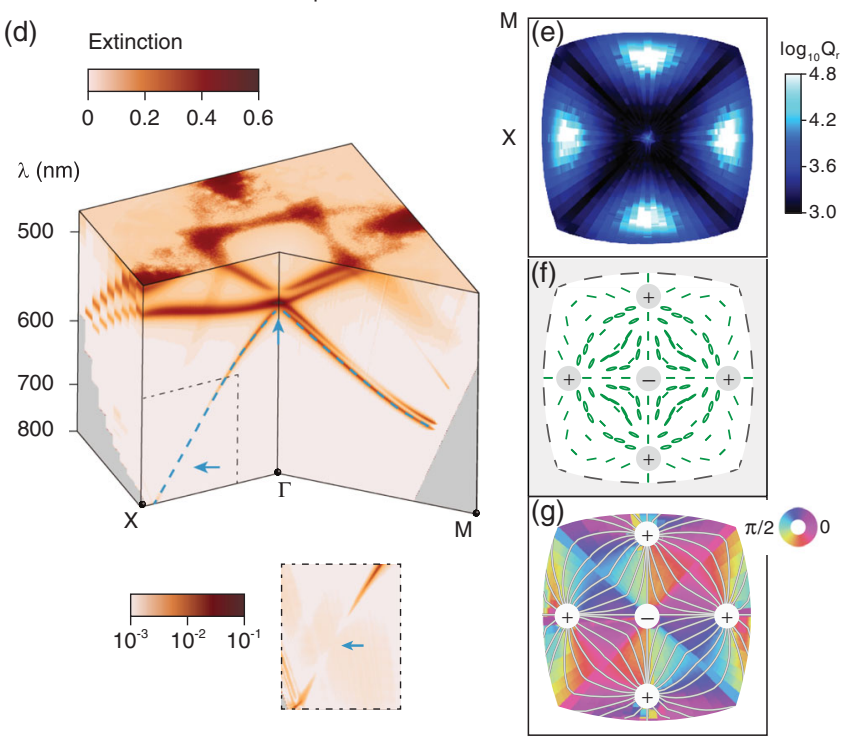

FIG. 2. (a) Schematic view of the experimental setup. BS, beam splitter; $L$, lens; $P$, polarizer. (b) SEM image of the sample. (c) Examples of polarization-dependent extinction spectra for three $\alpha$. Here, the $\Gamma-X$ direction of the sample is parallel to the slit of the spectrometer. (d) Measured band structure inside the FBZ. Vortices are marked with blue arrows, with a close-up on the bottom. (e) $Q$ factors of the lowest radiative band, as highlighted by the blue dashed lines in (d). It corresponds to the green line (band 2) in Fig. 1(a). (f) Measured polarization ellipses of band 2. Vortices and their topological charges are marked with \pm signs. (g) Measured angle distribution as the major axis of polarization states, represented by streamlines and colors.

and line segments above denote elliptical and linear polarizations, respectively. Figure $2(\mathrm{~g})$ shows the measured polarization vector distribution as the major axis of those elliptical polarization states in the whole FBZ, plotted as streamlines on top of a pseudocolor plot. Five polarization vortices are clearly observed with one -1 vortex at $\Gamma$ surrounded by four +1 vortices (related by the $C_{4 v}$ symmetry of the lattice). At the core of each vortex, the polarization is ill defined, indicating an absence of radiation and corresponding to the divergent $Q$ factors in Fig. 2(e). Because of their topological nature, polarization vortices are insensitive to the variation of the structure parameters such as filling fractions and thicknesses, which we verified 
experimentally in Supplemental Material [33]. The experimentally obtained topological charges are in good agreement with the theoretical prediction in Fig. 1(c).

For an even more direct visualization of the vortices in the FBZ, we use the second mode of operation and sum the isofrequency contours measured at a continuous range of wavelengths. We took a series of polarization-averaged isofrequency contours from 550 to $740 \mathrm{~nm}$ with a wavelength interval of $10 \mathrm{~nm}$. The bandwidth of the color filters is also $10 \mathrm{~nm}$, so the isofrequency contours overlap, and the whole wavelength range is covered. A few examples of the measured isofrequency contours are shown in Fig. 3(a) (the others are shown in Supplemental Material [33]). Note that only the colored contours with smaller wave vectors are from band 2, while those in light gray are from the higher bands. By summing the isofrequency contours contributed by band 2, we obtained the extinction data for the whole dispersion band and plotted it in the central figure in Fig. 3(c). Five dark regions are centered at the vortex cores with near-zero extinction in the momentum space, corresponding to the polarization vortices.

(a)

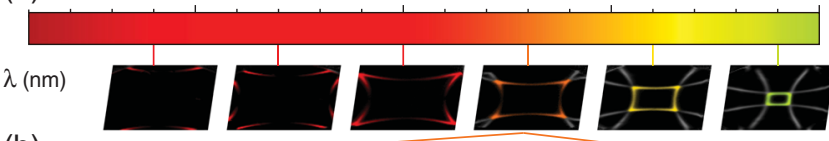

(b)

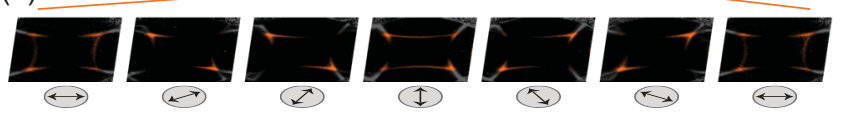

(c)

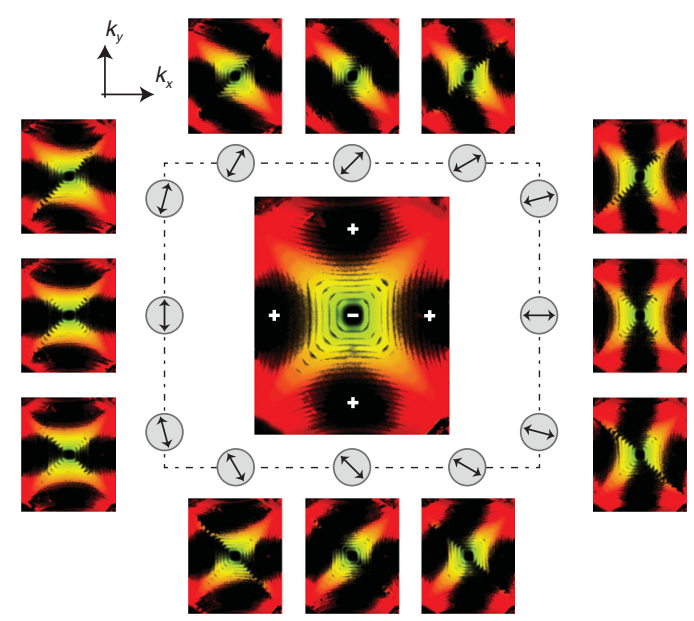

FIG. 3. (a) Examples of measured isofrequency contours under narrow-band illumination at wavelengths of 560, 590, 620, 650, 680 , and $710 \mathrm{~nm}$. Colored signals are from the band of interest, and light gray signals are from higher bands. (b) Examples of measured polarization-resolved isofrequency contours at $620 \mathrm{~nm}$. (c) Extinction map of the band of interest in the FBZ, obtained by summing 20 isofrequency contours. Different colors correspond to different wavelengths, with the color map shown in (a). Center plot: The polarization-averaged data. Outer plots: The polarization-resolved data; the arrows aside, the direction of the polarizer.
To directly resolve the winding of polarization around the vortices, we recorded the isofrequency contours as a function of the polarizer angle, shown in Fig. 3(b). The outer plots in Fig. 3(c) show the summed isofrequency contours at different polarizer angles; the signals from states with polarization vectors perpendicular to the polarizer nearly diminish, forming a dark strip around each vortex. Those dark strips spin with the polarizer. The spinning direction and speed of them directly reveal the sign and magnitude of the topological charges. Under the counterclockwise rotation of the polarizer, the spin direction of the vortex at the $\Gamma$ point is clockwise, while others spin counterclockwise. Consequently, they have opposite signs of charges. Since all five vortices rotate at the same speed as that of the polarizer, they have the same magnitude of charges of 1 . This direct observation of topological charges is consistent with the numerical results in Fig. 1(c) as well as the measured polarization distribution in Fig. 2(g). Among the key experimental results of this work, an animation showing these dark strips spinning with the polarizer is presented in Supplemental Material [33].

The topological charge of a vortex can be an arbitrary signed integer. So far, we have observed \pm 1 charges in the square lattice plasmonic crystal with $C_{4 v}$ point group symmetry. Higher-order charges can be realized in two ways: lattices with a higher symmetry group [7] or higher bands with a larger value of $n$. Here, we demonstrate both approaches.

To demonstrate the first approach, we fabricated a hexagonal lattice with $C_{6 v}$ symmetry and measured polarization-dependent isofrequency contours. As shown in the SEM image in Fig. 4(a), the structure is a 70-nm PMMA layer patterned with a hexagonal lattice on a flat silver substrate (periodicity, $600 \mathrm{~nm}$; hole diameter, $330 \mathrm{~nm}$ ). The measured band structure is shown in Fig. 4(a), while the calculation is shown in Supplemental Material [33]. Here, we picked the second band [shown as a blue dashed line in Fig. 4(a)] to observe vortices with high-order charges. The polarization distribution around the $\Gamma$ point measured are shown in the central right panel in Fig. 4(b). The topological charge of this central vortex is -2 . Away from $\Gamma$, there are six polarization vortices in the $\Gamma-K$ directions. In the polarization-resolved isofrequency contours [outer plots in Fig. 4(b)], the dark strips of these six outside vortices rotate counterclockwise with the same angular speed as that of the polarizer, corresponding to +1 charges. Meanwhile, the one at the $\Gamma$ point rotates clockwise with half the speed as that of the polarizer, corresponding to a vortex with -2 charge. The corresponding animation of these spinning patterns is shown in Supplemental Material [33]. Also, we fabricated a $C_{4 v}$ sample with a larger period to demonstrate the second approach. We found polarization vortices with a topological charge of -3 with $n=-1$ at the $M$ point of band 5. Details are shown in Supplemental Material [33]. 


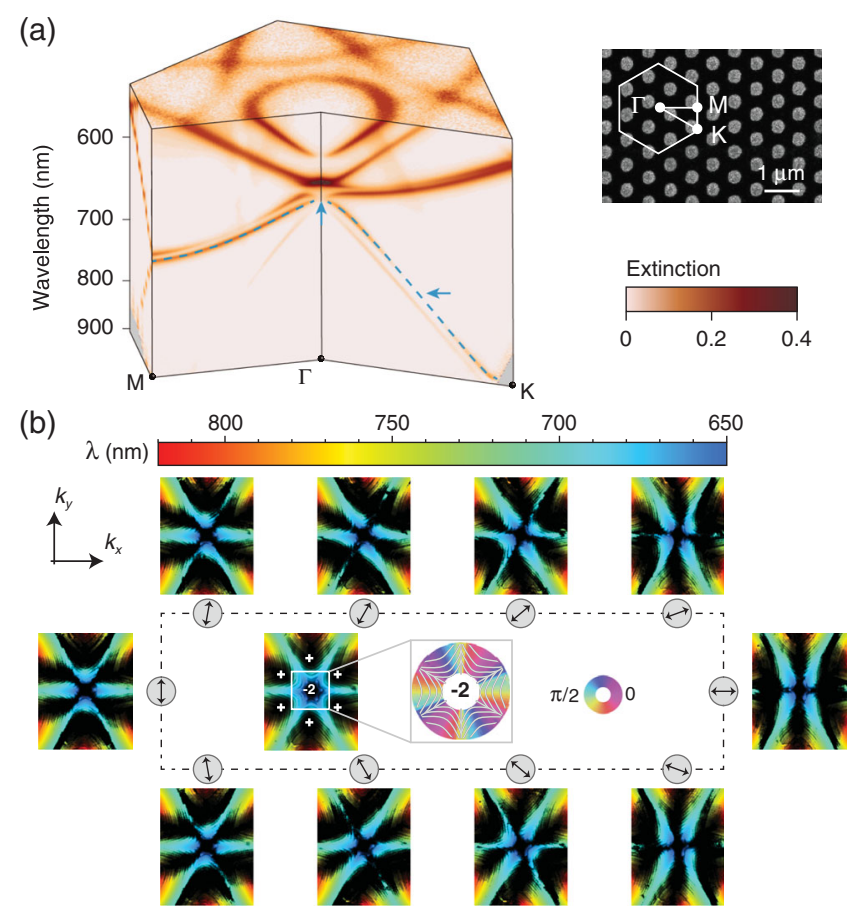

FIG. 4. (a) SEM image of the structure (right) and measured band structures using unpolarized illumination (left). Vortices are marked with blue arrows. (b) Extinction map of the band of interest [blue dashed line in (a)], obtained by summing 18 isofrequency contours. The colors correspond to different wavelengths, with the color map shown at the top. Central plots: Polarization-averaged data and measured distribution of polarization vectors near the $\Gamma$ point; outer plots: polarization-resolved data; arrows aside, direction of the polarizer.

In conclusion, we have experimentally observed the momentum-space polarization vortices in the entire FBZ of plasmonic crystals. Vortices with topological charges of \pm 1 and -3 were observed in a square lattice, and topological charges of -2 and +1 were observed in a hexagonal lattice. High-order vector beams could be generated at vortex cores of high-order momentum-space vortices, giving a promising way for on-chip applications on information encoding and optical tweezing. Such a phenomenon is generic for vector fields [45] and is an additional topological feature in band structures [26]. Transverse wave systems exhibiting polarization shall display this feature. Novel light-matter interactions could be studied by applying momentum-space polarization vortices.

We thank Chen Fang, Zhong Wang, Haiwei Yin, Shaoyu Yin, and Bo Zhen for helpful discussions. The work was supported by 973 and China National Key Basic Research Program (2015CB659400, 2016YFA0301100, 2016YFA0302000, 2017YFA0303800, and 2016YFA0302400) and National Science Foundation of China (11774063, 11727811, 11721404). The research of L. S. was further supported by Science and Technology Commission of Shanghai Municipality (17ZR1442300,
17142200100), State key laboratory of surface physics (ZA2014-01), Professor of Special Appointment (Eastern Scholar) at Shanghai Institutions of Higher Learning. L. S. and L. L. was also supported by the Recruitment Program of Global Youth Experts (1000 plans).

Y.Z., A. C., and W. L. contributed equally to this work.

*1shi@fudan.edu.cn

${ }^{\dagger}$ linglu@iphy.ac.cn

\#ji@ fudan.edu.cn

[1] N. D. Mermin, Rev. Mod. Phys. 51, 591 (1979).

[2] J. Nye and J. Hajnal, Proc. R. Soc. A 409, 21 (1987).

[3] R. W. Schoonover and T. D. Visser, Opt. Express 14, 5733 (2006).

[4] M. R. Dennis, K. O’Holleran, and M. J. Padgett, Prog. Opt. 53, 293 (2009).

[5] J. Kosterlitz and D. Thouless, J. Phys. C 5, L124 (1972).

[6] D. Hsieh et al., Science 323, 919 (2009).

[7] B. Zhen, C. W. Hsu, L. Lu, A. D. Stone, and M. Soljačić, Phys. Rev. Lett. 113, 257401 (2014).

[8] E. Miyai, K. Sakai, T. Okano, W. Kunishi, D. Ohnishi, and S. Noda, Nature (London) 441, 946 (2006).

[9] S. Iwahashi, Y. Kurosaka, K. Sakai, K. Kitamura, N. Takayama, and S. Noda, Opt. Express 19, 11963 (2011).

[10] C. W. Hsu, B. Zhen, A. D. Stone, J. D. Joannopoulos, and M. Soljačić, Nat. Rev. Mater. 1, 16048 (2016).

[11] D. C. Marinica, A. G. Borisov, and S. V. Shabanov, Phys. Rev. Lett. 100, 183902 (2008).

[12] E. N. Bulgakov and A. F. Sadreev, Phys. Rev. B 78, 075105 (2008).

[13] V. Liu, M. Povinelli, and S. Fan, Opt. Express 17, 21897 (2009).

[14] Y. Plotnik, O. Peleg, F. Dreisow, M. Heinrich, S. Nolte, A. Szameit, and M. Segev, Phys. Rev. Lett. 107, 183901 (2011).

[15] C. W. Hsu, B. Zhen, J. Lee, S.-L. Chua, S. G. Johnson, J. D. Joannopoulos, and M. Soljacic, Nature (London) 499, 188 (2013).

[16] G. Corrielli, G. Della Valle, A. Crespi, R. Osellame, and S. Longhi, Phys. Rev. Lett. 111, 220403 (2013).

[17] S. Weimann, Y. Xu, R. Keil, A. E. Miroshnichenko, A. Tünnermann, S. Nolte, A. A. Sukhorukov, A. Szameit, and Y. S. Kivshar, Phys. Rev. Lett. 111, 240403 (2013).

[18] Y. Yang, C. Peng, Y. Liang, Z. Li, and S. Noda, Phys. Rev. Lett. 113, 037401 (2014).

[19] M. G. Silveirinha, Phys. Rev. A 89, 023813 (2014).

[20] F. Monticone and A. Alu, Phys. Rev. Lett. 112, 213903 (2014).

[21] R. Gansch, S. Kalchmair, P. Genevet, T. Zederbauer, H. Detz, A. M. Andrews, W. Schrenk, F. Capasso, M. Lončar, and G. Strasser, Light Sci. Appl. 5, e16147 (2016).

[22] A. Kodigala, T. Lepetit, Q. Gu, B. Bahari, Y. Fainman, and B. Kanté, Nature (London) 541, 196 (2017).

[23] J. Gomis-Bresco, D. Artigas, and L. Torner, Nat. Photonics 11, 232 (2017).

[24] Y.-X. Xiao, G. Ma, Z.-Q. Zhang, and C. T. Chan, Phys. Rev. Lett. 118, 166803 (2017). 
[25] E. N. Bulgakov and D. N. Maksimov, Phys. Rev. Lett. 118, 267401 (2017).

[26] L. Lu, J. D. Joannopoulos, and M. Soljačić, Nat. Photonics 8, 821 (2014).

[27] D. Leykam, M. C. Rechtsman, and Y. D. Chong, Phys. Rev. Lett. 117, 013902 (2016).

[28] C. He, X. Sun, X. Liu, M. Lu, Y. Chen, L. Feng, and Y. Chen, Proc. Natl. Acad. Sci. U.S.A. 113, 4924 (2016).

[29] B. Yang, Q. Guo, B. Tremain, L. E. Barr, W. Gao, H. Liu, B. Béri, Y. Xiang, D. Fan, A. P. Hibbins, and S. Zhang, Nat. Commun. 8, 97 (2017).

[30] Y. Guo, M. Xiao, and S. Fan, Phys. Rev. Lett. 119, 167401 (2017).

[31] Q. Wang, M. Xiao, H. Liu, S. Zhu, and C. T. Chan, Phys. Rev. X 7, 031032 (2017).

[32] A. M. Yao and M. J. Padgett, Adv. Opt. Photonics 3, 161 (2011).

[33] See Supplemental Material at http://link.aps.org/ supplemental/10.1103/PhysRevLett.120.186103 for experimental methods, notes of temporal coupled-mode theory and supplemental figures.

[34] D. Han, F. Wu, X. Li, C. Xu, X. Liu, and J. Zi, Appl. Phys. Lett. 89, 091104 (2006).

[35] I. De Leon and F. S. Roux, Phys. Rev. B 71, 235105 (2005).
[36] See Supplemental Material at http://link.aps.org/ supplemental/10.1103/PhysRevLett.120.186103 for the derivation on the link between polarization states and polarization-dependent extinction spectra, which includes Refs. [7,37-44].

[37] C. W. Hsu, B. Zhen, M. Soljačić, and A. D. Stone, arXiv:1708.02197.

[38] J. Korger, T. Kolb, P. Banzer, A. Aiello, C. Wittmann, C. Marquardt, and G. Leuchs, Opt. Express 21, 27032 (2013).

[39] W. Suh, Z. Wang, and S. Fan, IEEE J. Quantum Electron. 40, 1511 (2004).

[40] S. Fan and J. D. Joannopoulos, Phys. Rev. B 65, 235112 (2002).

[41] S. Fan, W. Suh, and J. D. Joannopoulos, J. Opt. Soc. Am. A 20, 569 (2003).

[42] B. Zhen, C. Hsu, Y. Igarashi, L. Lu, I. Kaminer, A. Pick, S. Chua, J. Joannopoulos, and M. Soljačić, Nature (London) 525, 354 (2015).

[43] H. Zhou, B. Zhen, C. W. Hsu, O. D. Miller, S. G. Johnson, J. D. Joannopoulos, and M. Soljačić, Optica 3, 1079 (2016).

[44] L. Verslegers, Z. Yu, Z. Ruan, P. B. Catrysse, and S. Fan, Phys. Rev. Lett. 108, 083902 (2012).

[45] B.-J. Yang, M. S. Bahramy, and N. Nagaosa, Nat. Commun. 4, 1524 (2013). 